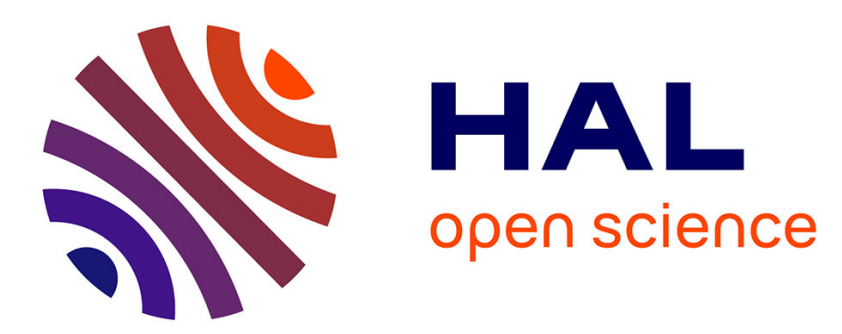

\title{
Ge-rich graded-index Si1-xGex racetrack resonators for long-wave infrared photonics
}

\author{
Joan Manel Ramirez, Vladyslav Vakarin, Qiankun Liu, Jacopo Frigerio, \\ Andrea Ballabio, Xavier Le Roux, Giovanni Isella, Carlos Alonso-Ramos, \\ Miguel Montesinos, Laurent Vivien, et al.
}

\section{To cite this version:}

Joan Manel Ramirez, Vladyslav Vakarin, Qiankun Liu, Jacopo Frigerio, Andrea Ballabio, et al.. Ge-rich graded-index Sil-xGex racetrack resonators for long-wave infrared photonics. Quantum Sensing and Nano Electronics and Photonics XVI, Feb 2019, San Francisco, United States. $10.1117 / 12.2510738$. hal-02362474

\section{HAL Id: hal-02362474 https://hal.science/hal-02362474}

Submitted on 13 Nov 2019

HAL is a multi-disciplinary open access archive for the deposit and dissemination of scientific research documents, whether they are published or not. The documents may come from teaching and research institutions in France or abroad, or from public or private research centers.
L'archive ouverte pluridisciplinaire HAL, est destinée au dépôt et à la diffusion de documents scientifiques de niveau recherche, publiés ou non, émanant des établissements d'enseignement et de recherche français ou étrangers, des laboratoires publics ou privés. 


\title{
Ge-rich graded-index $\mathrm{Si}_{1-\mathrm{x}} \mathrm{Ge}_{\mathrm{x}}$ racetrack resonators for long-wave infrared photonics
}

\author{
J. M. Ramirez ${ }^{\mathrm{a}, \mathrm{b}}$, V. Vakarin a, Q. Liu ${ }^{\mathrm{a}}$, J. Frigerio ${ }^{\mathrm{c}}$, A. Ballabio ${ }^{\mathrm{c}}$, X. Le Roux ${ }^{\mathrm{a}}$, G. Isella ${ }^{\mathrm{c}}$, C. Alonso- \\ Ramos $^{\mathrm{a}}$, M. Montesinos ${ }^{\mathrm{a}}$, L. Vivien ${ }^{\mathrm{a}}$ and D. Marris-Morini ${ }^{\mathrm{a}}$ \\ ${ }^{a}$ Centre de Nanosciences et de Nanotechnologies, Université Paris Sud, CNRS, Université Paris

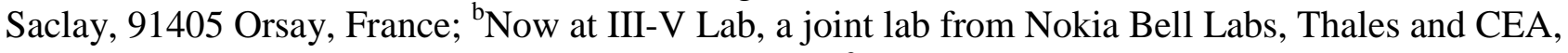 \\ 1 avenue Augustin Fresnel, 91767 Palaiseau Cedex ; ${ }^{c}$ L-NESS, Dipartimento di Fisica, Politecnico \\ di Milano, Polo di Como, Via Anzani 42, 22100 Como, Italy
}

\begin{abstract}
Mid-infrared racetrack resonators are demonstrated working at $8 \mu \mathrm{m}$ wavelength. The devices are based on a graded SiGe platform providing low propagation loss on a large wavelength range in the mid-IR. Different resonators designs have been fabricated, with varying gap distances in the directional coupler. Q factors of more than 3000 have been experimentally demonstrated. These results pave the way towards compact mid-IR sensors or efficient active devices.
\end{abstract}

Keywords: Photonic integrated circuits, resonator, mid-infrared, silicon photonics

\section{INTRODUCTION}

The mid-IR wavelength range covering from $2 \mu \mathrm{m}$ to $20 \mu \mathrm{m}$ has raised as a promising 'sweet spot' to develop advanced silicon photonic systems [1-4] owing to its several interesting features, including: i) The low material dispersion present on Si-based materials in the mid-IR wavelength range, which eases the design of dispersion-engineered photonic integrated circuits with broadband flat dispersion windows; ii) the absence of two-photon absorption losses, which are known to be detrimental for certain applications, notably for components requiring high-power management; iii) the mid-IR wavelength range allocates a large number of 'molecular fingerprints' associated with vibrational modes of many substances and molecules relevant for the human being, thus being of interest for the implementation of ultra-sensitive optical sensors with potentially parts-per-billion floor detection [5]. Moreover, the mid-IR spectrum also provides two atmospheric transparency windows placed at 3-5 $\mu \mathrm{m}$ and 8-13 $\mu \mathrm{m}$ respectively that can be leveraged to implement novel free-space optical communication systems when combined with high power quantum cascade lasers [6]. To successfully implement such interesting functionalities, a material platform compatible with the CMOS processing while having a large transparency window is required. In this regard, Ge stands as one of the most promising materials as it fulfils both conditions. With a transparency window that spans from the near infrared up to a wavelength of $\lambda=15 \mu \mathrm{m}$, Ge provides a unique opportunity to exploit the long-wave infrared range [7]. An interesting family of the Ge-based platform is the one that uses an epitaxial SiGe graded buffer directly grown on $\mathrm{Si}$, as they offer fine control of the optical properties by tuning the graded Ge profile in the growth direction to form the waveguide core. Moreover, this strategy enables a gradual accommodation of the lattice mismatch between $\mathrm{Si}$ and $\mathrm{Ge}$, providing a low-defect density alloy with low propagation losses [8]. Various demonstrations of mid-IR photonic components based on graded SiGe waveguides have been reported, including ultra-broadband Mach Zehnder interferometers [9] and integrated Fourier-transform spectrometers [10]. Furthermore the ability of this platform to sense small concentrations of methane gas has been discussed [11] while non-linear properties of Ge rich SiGe alloys have been studied as a function of Ge concentration [12], opening the path to the design of efficient devices based on NL effects such as supercontinuum generation though self-phase modulation [13].

In this context, integrated resonators appear as important building blocks to be developed, as they can be used (i) to develop compact sensors by an increased interaction length between the propagating mode and the analyte, (ii) as an interferometric structure for next development of optical modulators, (iii) as a nonlinear device, to provide a strong-light matter interaction with the nonlinear material. Although ring resonators have been largely developed using Silicon-OnInsulator waveguides in the near-IR wavelength range [14], their extension towards mid-IR wavelengths using graded 
SiGe waveguides is not straightforward. Indeed a reduced mode confinement could prevent from compact bends and thus limiting achievable Free Spectral Ranges (FSR).

In this paper we report on the main design rules and the device characterization showing that by carefully designing the coupler and ring resonator we have been able to demonstrate for the first time an integrated mid-IR racetrack ring resonator working at $8 \mu \mathrm{m}$ wavelength, with a maximum loaded quality factor (Q) of 3200 . This result paves the way towards efficient non-linear devices and compact sensors in the long-wave infrared (LWIR).

\section{DESIGN OF THE GE-RICH GRADED-INDEX SI $_{1-X} \mathbf{G E}_{X}$ RACETRACK RESONATORS}

\subsection{Ge-rich SiGe racetrack resonator design and fabrication.}

The Ge-rich SiGe waveguides are based on the epilayer reported in Fig 1(a). The 6- $\mu$ m thick graded SiGe waveguide core is grown on a standard Si substrate using Low Energy Plasma Enhanced Chemical Vapor Deposition (LEPECVD). This approach allows an efficient reduction of the number of threading dislocations (TDD) by gradual accommodation of the Si-Ge lattice mismatch in the layer stack. In addition the linear refractive index increases (as shown in Fig. 1(b)) due to the gradual increase of the Ge content in SiGe alloy, allowing the confinement of light at the top of the graded SiGe layer. Rib waveguides can then been defined by etching the SiGe graded layer as shown in Fig 1(a). Directional couplers and racetrack resonators have been designed using numerical simulations as illustrated in Fig 1.c. After optimization, the coupler length has been fixed to $200 \mu \mathrm{m}$ while gap distances varying from 0.6 to $1.2 \mu \mathrm{m}$ have been selected. The ring radius $\mathrm{R}$ in the racetrack is fixed to $250 \mu \mathrm{m}$ to avoid bending losses.

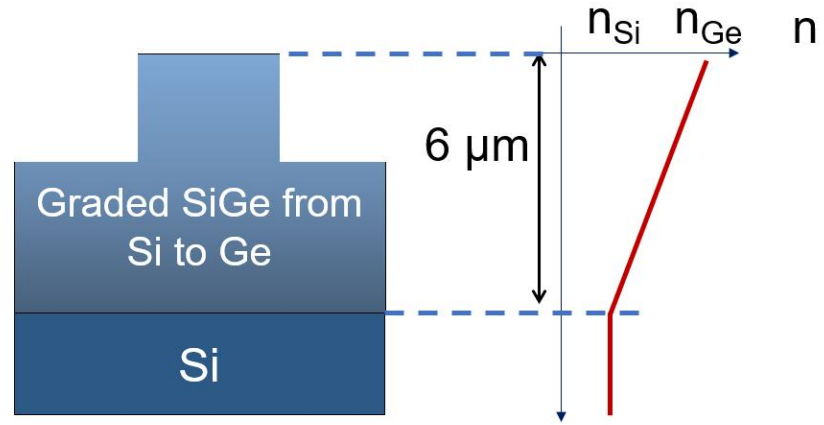

(a)

(b)

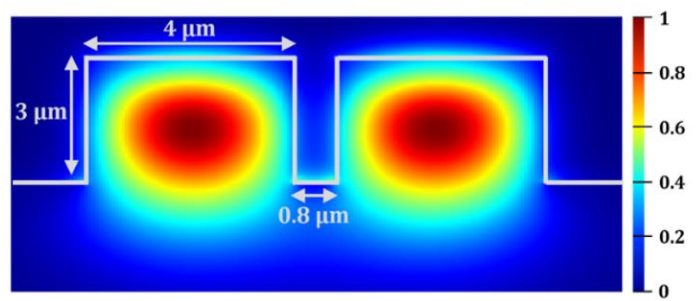

(c)

Figure 1. Cut-view of the Ge-rich SiGe waveguides (a): epilayer grown by LEPECVD in standard Si substrate. (b) : refractive index profile along the growth direction; (c) example of super-mode calculation in the directional coupler. 
The racetrack ring resonators were fabricated using e-beam lithography and ICP etching. A scanning electron microscopy (SEM) image of the racetrack resonator is shown in Fig 2(a). After fabrication, waveguide facets were diced for butt-coupling using a free-space experimental set-up.

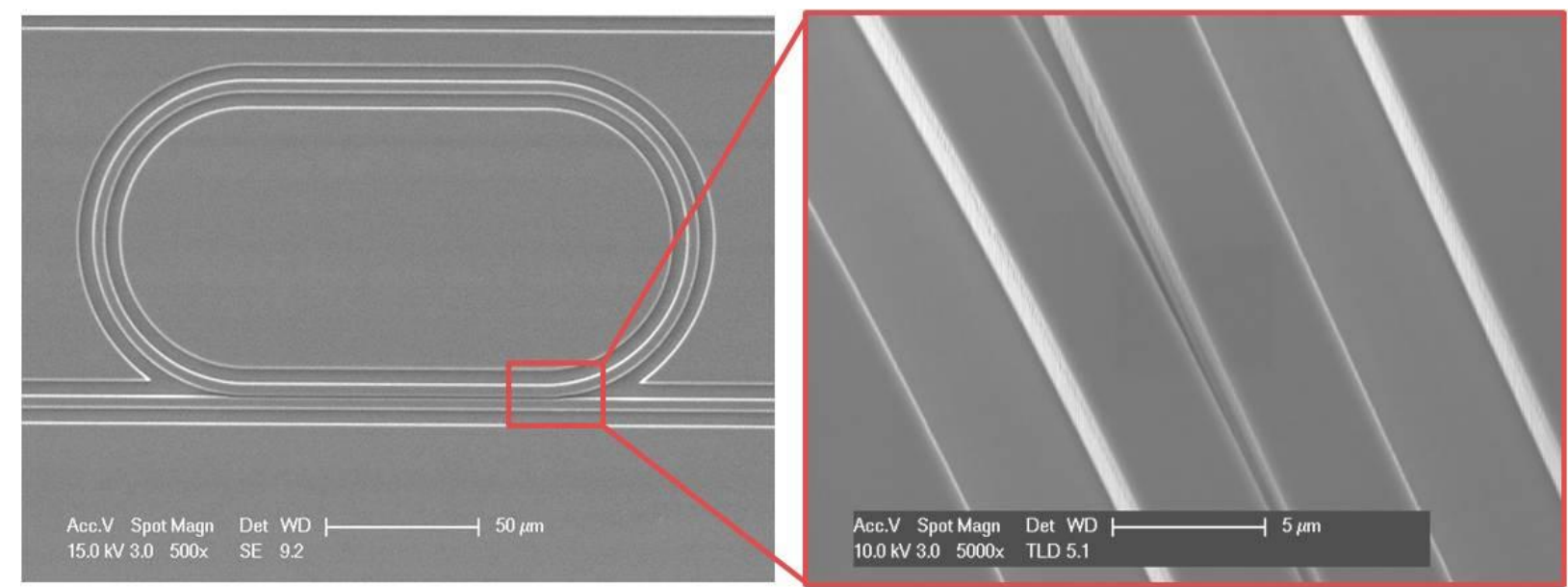

Figure 2: Top view SEM image of a single racetrack resonator with the access bus waveguide. The figure at the right-hand side shows a zoomed-in area of the coupling region.

\subsection{Characterization set-up.}

Measurements were performed using an ad-hoc free-space mid-IR setup, placed inside an isolation box and equipped with a dry air filling system, to reduce the impact of the atmospheric absorption (Fig. 3(b)). Transmission measurements were performed using a mid-IR tunable external cavity quantum cascade laser (MIRCAT) from $\lambda=7.5 \mu \mathrm{m}\left(1333.3 \mathrm{~cm}^{-}\right.$ ${ }^{1}$ ) to $8.6 \mu \mathrm{m}\left(1162.8 \mathrm{~cm}^{-1}\right)$, operating in pulsed regime (duty cycle of $5 \%$ and repetition rate of $100 \mathrm{kHz}$ ), with a linewidth specified to be below $1 \mathrm{~cm}^{-1}$. Input/output chip butt-coupling was carried out by means of aspheric $\mathrm{ZnSe}$ lenses. The collected signal was sent to either an MCT detector or a mid-IR camera (see Fig 3. (a)).

\section{Free-space Optical (FSO) system}
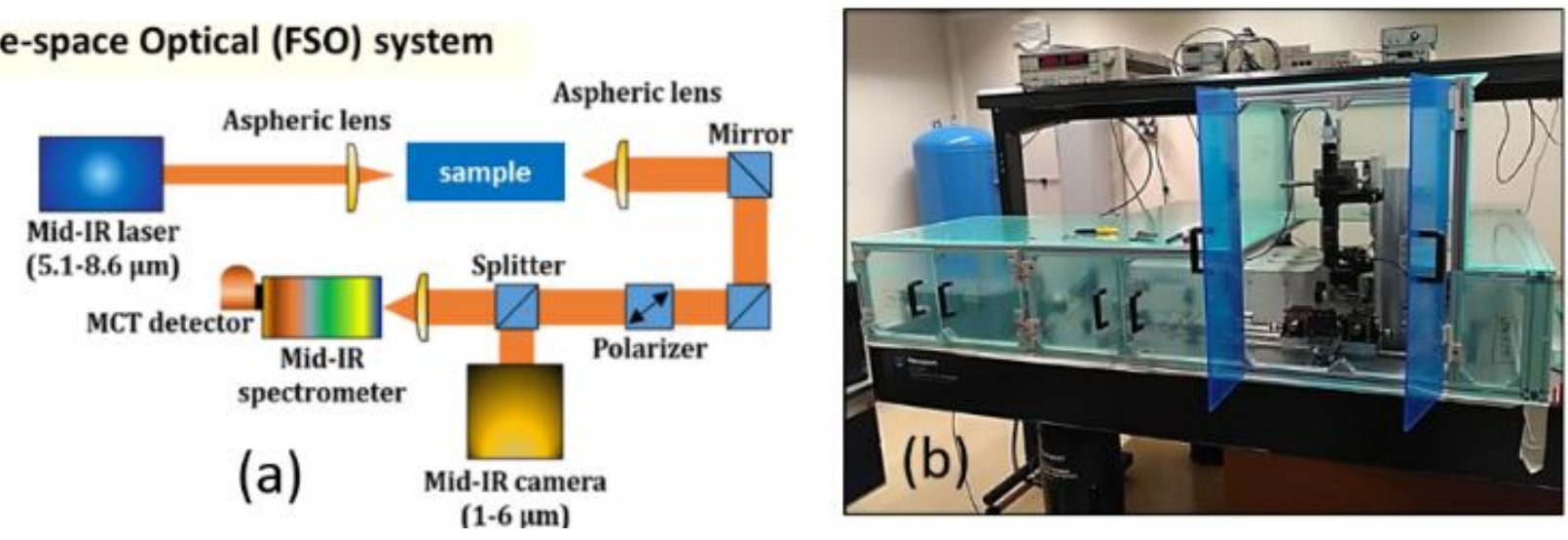

Figure 3: (a) Schematic view of the experimental set-up used for the mid-IR characterization: (b) Picture of the experimental set-up. 


\subsection{Experimental results}

Figure 4 summarizes the normalized quasi-TM transmission of the racetrack ring resonators for the different gap distances. Resonances are clearly observed in all the reported results. As expected, the critical coupling condition shifts towards larger wavelength (lower wavenumber) when the gap distance is increased, corresponding to a decrease of the intensity coupling in the directional coupler. From the measurements, a linearly increasing FSR with wavelength can be deduced for the different racetrack resonators, as expected. This trend is reported in Fig 5. A good correlation between measured FSR and calculation confirms the single mode operation of the devices. Interestingly, a maximum loaded quality factor of $\mathrm{Q} \approx 3200$ is obtained in the different configurations.

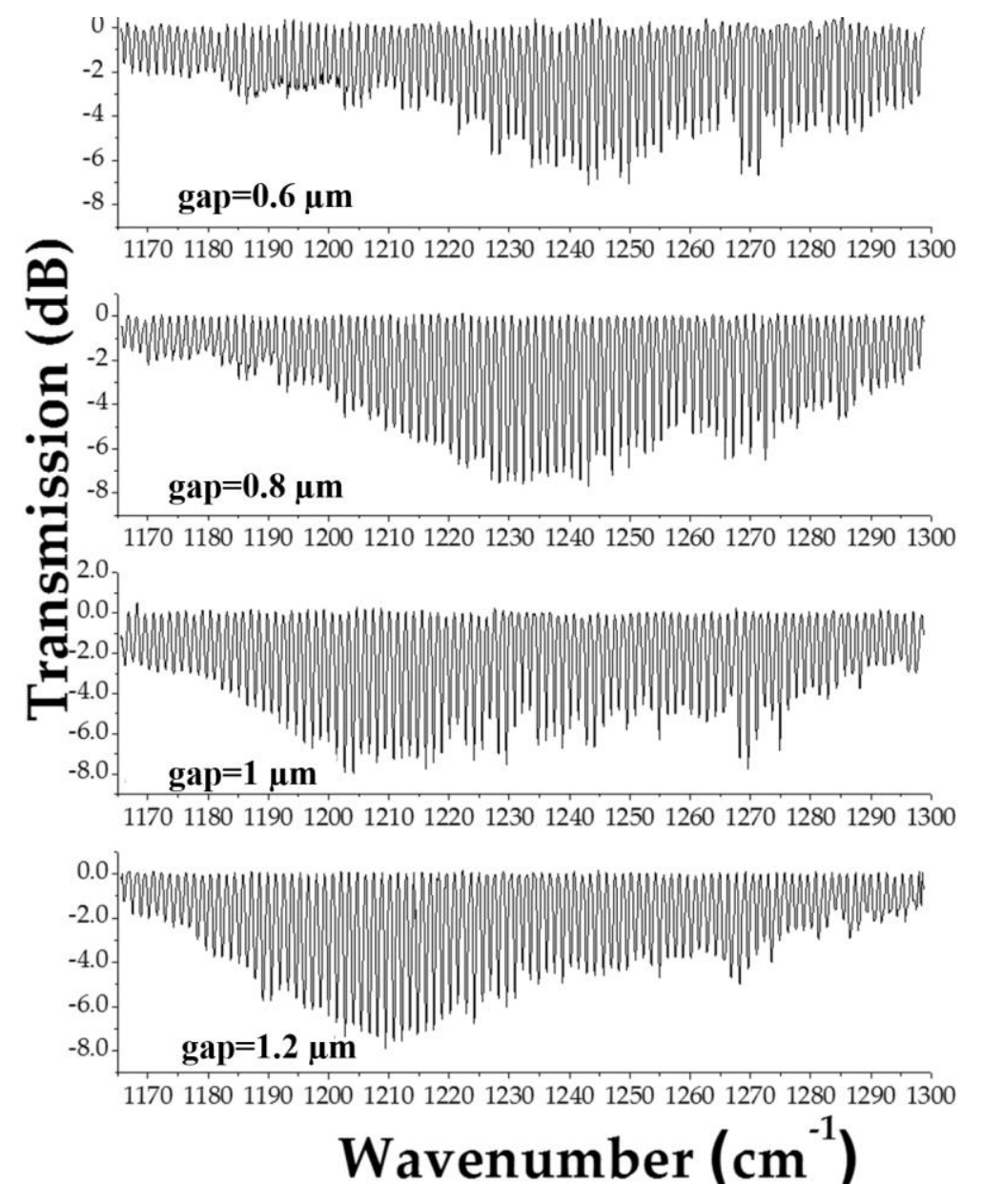

Figure 4: . Experimental transmission spectra of racetrack ring resonators operating in quasi-TM polarization, for different values of the gap within the directional coupler, from 0.6 to $1.2 \mu \mathrm{m}$. The shift of the critical coupling condition related to a decrease of the intensity coupled in the racetrack when the gap is increased is clearly seen. 


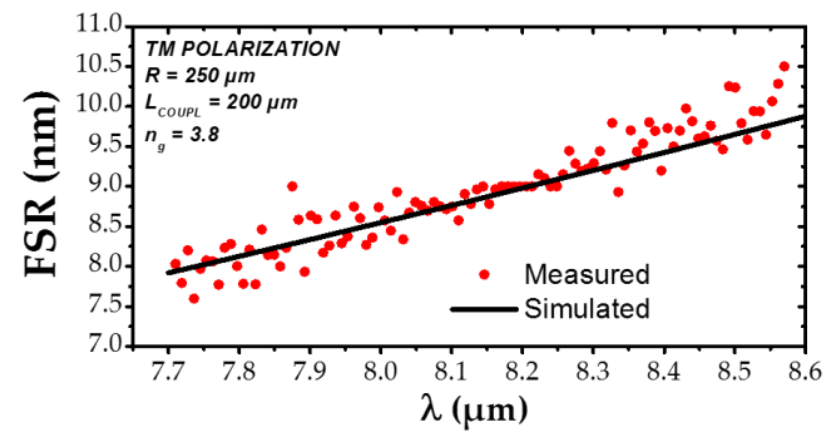

Figure 5: Free Spectral Range extracted from the measurement and compared with theoretical value. A good correlation is obtained indicating the single mode operation of the racetrack ring resonators.

\section{CONCLUSION}

In conclusion, this paper reports the first demonstration of mid-IR racetrack resonators working around $8 \mu \mathrm{m}$ wavelength. The resonant structures are based on a $6 \mu \mathrm{m}$-thick graded SiGe platform. A maximum Q-factor of 3200 is demonstrated. This work paves the way towards the implementation of resonant structures for multi-target molecular spectroscopic sensors or for the development of non-linear devices in integrated photonic platforms.

\section{ACKNOWLEDGMENT.}

This project has received funding from the European Research Council (ERC) under the European Union's Horizon 2020 research and innovation program (grant agreement $\mathrm{N}^{\circ} 639107-$ INsPIRE).

\section{REFERENCES}

[1] Zhang L, Agarwal A M, Kimerling L C and Michel J. Nonlinear Group IV photonics based on silicon and germanium: from near-infrared to mid-infrared. Nanophotonics (2014). 3(4-5); 247-268.

[2] Nedeljkovic M, Khokhar A Z, Hu Y, Chen X, Soler Penades J, Stankovic S, Chong H M H, Thomson D J, Gardes F Y, Reed G T, Mashanovich G Z. Silicon photonic devices and platforms for the mid-infrared. Optical Materials Express (2013). 3 (9); 1205.

[3] Lin H, Luo Z, Gu T, Kimerling L C, Wada K, Agarwal A and Hu J. Mid-infrared integrated photonics on silicon: a perspective. Nanophotonics (2018). 7(2); 393-420.

[4] D. Marris-Morini, V. Vakarin, J-M. Ramirez, Q. Liu, A. Ballabio, J. Frigerio, M. Montesinos, C. Alonso-Ramos, X. Le Roux, S. Serna, D. Benedikovic, D. Chrastina, L. Vivien and G. Isella, Germanium-based integrated photonics from near- to mid-infrared applications Nanophotonics, (2018).

[5] J. Haas, B. Mizaikoff, “Advances in mid-infrared spectroscopy for chemical analysis," Annual Review of Analytical Chemistry, 9,45 (2016).

[6] X. Pang, O. Ozolins, R. Schatz, J. Storck, A. Udalcovs, J. Navarro, A. Kakkar, G. Maisons, M. Carras, G. Jacobsen, S. Popov, and S. Lourdudoss, "Gigabit free-space multi-level signal transmission with a mid-infrared quantum cascade laser operating at room temperature," Opt. Lett. 42, 3646-3649 (2017).

[7] R. Soref, "Mid-infrared photonics in silicon and germanium," Nature Photonics, 4, 495 (2010).

[8] J. M. Ramirez, Q. Liu, V. Vakarin, J. Frigerio, A. Ballabio, X. Le Roux, D. Bouville, L. Vivien, G. Isella and D. Marris-Morini, "Graded SiGe waveguides with broadband low-loss propagation in the mid infrared," Opt. Express, 26, 2, 870 (2018).

[9] V. Vakarin, J-M. Ramirez, J. Frigerio, A. Ballabio, X. Le Roux, Q. Liu, D. Bouville, L. Vivien, G. Isella, D. MarrisMorini, Ultra-wideband Ge-rich silicon germanium integrated Mach-Zehnder interferometer for mid-infrared spectroscopy, Optics Letters Vol. 42 n¹7, 3482-3485 (2017) 
[10] Q. Liu, J.M. Ramirez, V. Vakarin, X. Le Roux, C. Alonso-Ramos, J. Frigerio, A. Ballabio, E. Talamas Simola, D. Bouville, L. Vivien, G. Isella, D. Marris-Morini, Integrated broadband dual-polarization Ge-rich SiGe mid-infrared Fourier-Transform spectrometer, Optics Letters, 43 (20), 5021-5024 (2018).

[11] Q. Liu, J. Manel Ramirez, V. Vakarin, X. Le Roux, A. Ballabio, J. Frigerio, D. Chrastina, G. Isella, D. Bouville, L. Vivien, C. Alonso Ramos, D. Marris-Morini,Mid-infrared sensing between 5.2 and $6.6 \mu \mathrm{m}$ wavelengths using Gerich SiGe waveguides [Invited], Optical Materials Express, 8 (5), 1305 (2018)

[12] S. Serna, V. Vakarin, J.-M. Ramirez, J. Frigerio, A. Ballabio, X. Le Roux, L. Vivien, G. Isella, E. Cassan, N. Dubreuil, D. Marris-Morini, Nonlinear Properties of Ge-rich Si1-xGex Materials with Different Ge Concentrations, Scientific reports, 7, 14692 (2017)

[13] J .M. Ramirez, V. Vakarin, J. Frigerio, P. Chaisakul, D. Chrastina, X. Le Roux, A. Ballabio, L. Vivien, G. Isella, D. Marris-Morini, Ge-rich graded-index Sil-xGex waveguides with broadband tight mode confinement and flat anomalous dispersion for nonlinear mid-infrared photonics, Optics Express 25 (6) 6561 (2017) .

[14] W. Bogaerts, P. De Heyn, T. Van Vaerenbergh, K. De Vos, S. Kumar Selvaraja, T. Claes, P. Dumon, P. Bienstman, D. Van Thourhout, R. Baets, Silicon microring resonators, Laser Photonics Rev. 6 (1),47-73 (2012) 